\title{
Differences in Colony Morphology and Carbohydrate Fermentation of Clostridium sporogenes
}

\author{
By T. J. T. PRINCEWILL* \\ Department of Microbiology, The University, Leeds LS2 $9 J T$
}

(Received 12 April 1978; revised 3 May 1978)

\section{INTRODUCTION}

The first detailed description of Clostridium sporogenes was given by Metchnikoff (1908) who isolated the organism from faeces of normal individuals and from diarrhoeal material of patients with chronic colitis. Subsequently, different names were given to organisms with descriptions similar to that of $C$. sporogenes. Thus, organisms labelled 'Reading' bacillus (Donaldson \& Joyce, 1917; Donaldson, 1918; Harris, 1919); C. parasporogenes (McIntosh, 1917); C. tyrosinogenes (Hall, 1921, cited by Kahn, 1924); C. flabelliferum (Sturges \& Reddish, 1925); C. sporogenes var. A.P. Marie (Marie, 1925); and C. sporogenes var. equine (Prévot, 1938) are now classified in the $C$. sporogenes complex.

In the present study colonial morphology and sugar fermentation properties of C. sporogenes were examined to throw light on the variation found in the taxon.

\section{METHODS}

Cultures. Details of the 84 strains of $C$. sporogenes and their sources are given in Table 1. Lyophilized cultures were reconstituted, grown in cooked meat medium (CMM; Southern Group Laboratory, Hither Green Hospital, London SE13) and plated on fresh horse blood agar. After incubation at $37^{\circ} \mathrm{C}$ for $48 \mathrm{~h}$, single colonies were isolated and their identity was checked by routine methods.

Colony morphology. The test strains were grown on fresh blood, chocolate and egg-yolk agars and the colonial characters were recorded after incubation at $37^{\circ} \mathrm{C}$ for $48 \mathrm{~h}$.

Carbohydrate fermentation. Strains were examined for their ability to ferment glucose, glycerol, lactose, maltose, salicin, sucrose and starch in a modified MRS (de Man et al., 1960) medium, in which the peptone content was decreased to $1.25 \mathrm{mg} \mathrm{ml}^{-1}$, the $\mathrm{pH}$ indicator was phenol red and the $\mathrm{pH}$ was re-adjusted to $7 \cdot 2$. The medium was dispensed into test tubes containing Durham tubes and the appropriate Seitz-filtered carbohydrates were added to the sterile medium to give a final concentration of $1 \%$. Inocula were prepared by growing strains for $24 \mathrm{~h}$ at $37^{\circ} \mathrm{C}$ in MRS medium without peptone. The seeded sugars were incubated in anaerobe jars at $37^{\circ} \mathrm{C}$ and examined for fermentation reactions after $48 \mathrm{~h}$.

\section{RESULTS}

\section{Colony morphology}

The colony morphology of strains grown on fresh blood agar varied considerably. Generally, the colonies were large and flat with a rhizoid margin and a raised centre which was usually smooth and translucent and varied from pinpoint to 2 to $3 \mathrm{~mm}$ in diameter. The rest of the colony was dry and rough (Fig. 1 a). Some of the colonies were evenly smooth and flat with crenated edges and a matt surface showing a greyish to creamy white colour, but very occasionally small creamy white colonies with radial ridges and crenated edges or smooth translucent colonies with entire edges were seen (Fig. 1b,c).

Colonies on chocolate agar were similar to those on fresh blood agar but there was a

* Present address: Nigerian Institute for Trypanosomiasis Research, Vom, Via Jos, Nigeria. 
Fresh blood agar
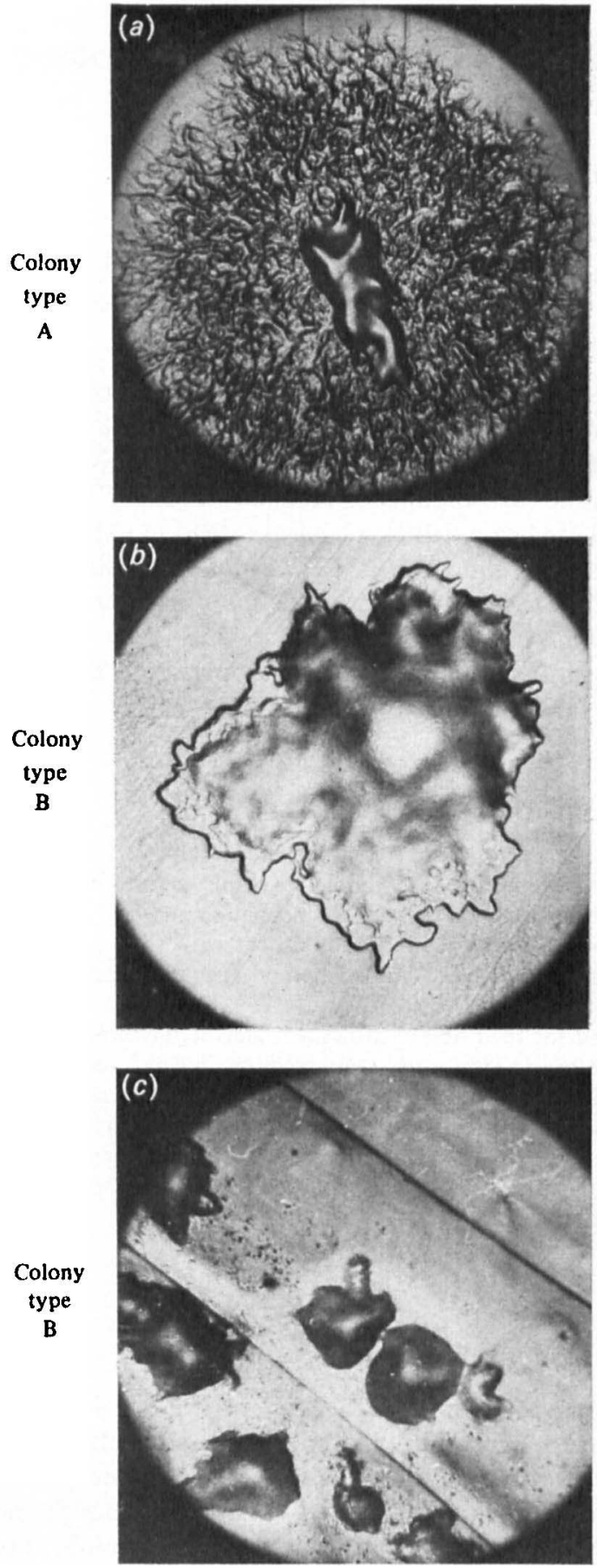

Egg-yolk agar
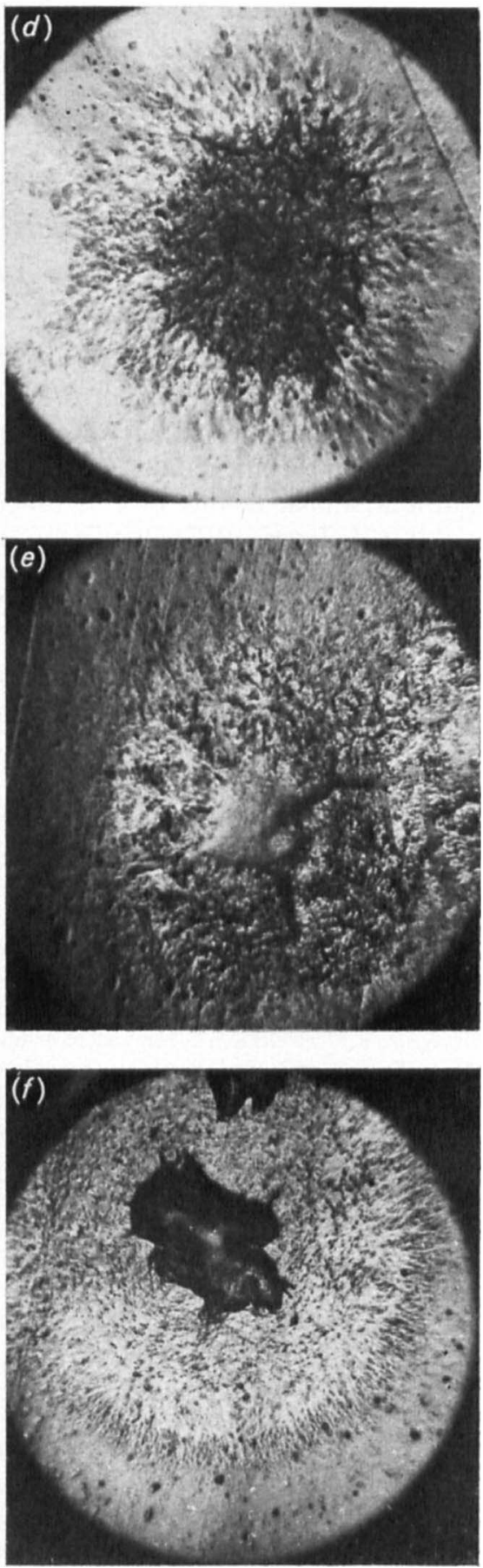

Fig. 1. Clostridium sporogenes: $(a, b, c)$ colonies on fresh blood agar of types A (strain 2$)$ and B (strains 59 and 60), respectively; $(d, e, f)$ colonies on egg-yolk agar of types A (strain 2) and B (strains 59 and 60 ), respectively. 
Table 1. Correlation between colony type and fermentation of sucrose and salicin by Clostridium sporogenes

\begin{tabular}{lrr} 
& \multicolumn{2}{c}{ Colony type } \\
Total no. of strains* & 66 & 18 \\
No. of strains producing & & \\
acid and gas in: & & \\
Salicin & 1 & 17 \\
Sucrose & 1 & 14 \\
Glucose & 66 & 18 \\
Maltose & 66 & 18 \\
Glycerol & 61 & 18 \\
Starch & 7 & 1 \\
Lactose & 0 & 0
\end{tabular}

* Of the 84 strains' of $C$. sporogenes, 7 were from this laboratory; 6 from the National Collection of Industrial Bacteria (NCIB), Torry Research Station, Aberdeen; 37 from L. S. McClung, Indiana University, U.S.A.; 9 from Wellcome Research Laboratories, Beckenham; 16 from A. Prévot, Pasteur Institute, Paris, France; 4 from the late H. Meisel, Serum Research Institute, Warsaw, Poland; and 5 from G. C. Mead, Food Research Institute, Norwich.

greater tendency towards rhizoid formation and the production of a brown pigmentation, the latter intensifying under aerobic conditions at room temperature.

Morphologically, the colonies on egg-yolk agar were of two main types with type A colonies more frequent than type B (Table 1). The type A colonies were flat, rhizoid, usually circular, with a feathery edge and a small, raised, somewhat dome-shaped or irregular centre; the rhizoid or arborescent appear ance gave the impression of a tendency to spread (Fig. $1 d$ ). In contrast, the type B colonies were entire with a raised centre and a very short rhizoid or feathery periphery. The central portion sometimes looked like a plateau with a raised margin and the body of the colonies occasionally showed radial ridges particularly when the raised centre was pyramidal (Fig. 1e,f).

\section{Carbohydrate fermentation}

All of the strains fermented glucose and maltose, most fermented glycerol ( $>90 \%$ but not starch, and none attacked lactose (Table 1). The results with sucrose and salicin were the most interesting. Thus, only one (strain 17) of the 15 strains that fermented sucrose and one (strain 19) of the 18 strains that fermented salicin did not produce a colony of type B (Table 1). Of the 18 strains which gave rise to type B colonies, 14 fermented both sucrose and salicin, three fermented salicin but not sucrose and only one failed to ferment either salicin or sucrose. The 64 strains with no action on sucrose and salicin and strains 17 and 19 all gave type A colonies on egg-yolk agar.

\section{DISCUSSION}

The colonial morphology of $C$. sporogenes on both fresh blood and chocolate agars is not sufficiently stable to be used in classification and identification. The work of Reed (1945) on the interconversion of smooth and rough forms vividly illustrates the futility of using colonial morphology alone in taxonomy (see Fig. 1); some workers (Holdeman \& Moore, 1975) have gone further by not using colonial characters at all in the classification of clostridia. McClung \& Toabe (1947) were the first to use egg-yolk agar as a differential medium, with which they achieved presumptive identification of some species of Clostridium. In the present study, the $C$. sporogenes strains exhibited two types of colony, A and B, on egg-yolk agar and this separation was supported by the results obtained in the 
fermentation of salicin and sucrose. Nakamura et al. (1977), using blood agars supplemented with glucose and brain heart infusion, found a cortelation between colony type and deoxyribonucleic acid homology in a study of $C$. sporogenes and $C$. botulinum strains. Their types I and II colonies compare with types $\mathrm{A}$ and $\mathrm{B}$.

In the present investigation, no attempt was made to study the relationship between strains of $C$. sporogenes and the proteolytic strains of $C$. botulinum. However, in a study on the specificity of somatic antisera for detecting C. botulinum, Lynt et al. (1972) included $C$. sporogenes strains and, although they observed differences in the physiological characteristics of the two groups of organisms, the differences were confined to the fermentation of a few sugars and colonial morphology on liver-veal-egg-agar. These workers concluded that there was no regular pattern by which $C$. sporogenes could be separated from $C$. botulinum on the basis of sugar fermentation and colony morphology.

Fermentation reactions are helpful in the taxonomy of Clostridium. In the present study, all the $C$. sporogenes strains produced acid and gas from glucoseand maltose but did not ferment lactose. However, these results are not in complete agreement with those of Holdeman \& Moore (1975) who recorded a weak fermentation of glucose and a negative or weak reaction in maltose by most strains of $C$. sporogenes. Most $C$. sporogenes strains fail to ferment sucrose and salicin and produce type A colonies on egg-yolk agar and can be differentiated from those that ferment the sugars and exhibit type B colonies (Table 1). Sucrose and salicin fermentation are also important in the differentiation of other Clostridium species (McIntosh, 1917; Robertson, 1918); C. septicum ferments salicin and not sucrose whereas C. chauvoei does the reverse (Moussa, 1956; Princewill, 1969).

The basal medium used in the fermentation tests gave reproducible results. Clostridium sporogenes is a strongly proteolytic organism and so turns protein media alkaline due to deamination of the amino acids formed during proteolysis. Excessive proteolysis will therefore tend to mask acid production. By decreasing the peptone concentration to a minimum, acid production from sugars has been readily detected using phenol red as indicator. Measurement of culture $\mathrm{pH}$ with a $\mathrm{pH}$ meter would probably be more accurate but is not a practicable proposition in routine laboratories where large numbers of cultures have to be examined.

This work was supported in part by a scholarship awarded by the Nigerian Federal Ministry of Education. The author also wishes to thank $\mathrm{Mr}$ J. Wolf for suggesting the problem and for his invaluable advice and help throughout this investigation.

\section{REFERENCES}

DoNALDSON, R. (1918). Characters and properties of the 'Reading' bacillus, on which a new method of treatment of wounds has been based. Journal of Pathology and Bacteriology 22, 129151.

Donaldson, R. \& Joyce, J. L. (1917). A method of wound treatment by the introduction of living cultures of spore-bearing anaerobe of the proteolytic group. Lancet 2, 445-452.

Harris, J. E. C. (1919). Contributions to the biochemistry of pathogenic anaerobes. VII. The biochemical comparison of micro-organisms by quantitative methods. Journal of Pathology and Bacteriology 23, 30-49.

Holdeman, L. V. \& Moore, W. E. C. (1975). Anaerobic Laboratory Manual, 3rd edn. Blacksburg, Virginia: Virginia Polytechnic Institute and State University.
KAHN, M. C. (1924). Anaerobic spore-bearing bacteria of human intestine in health and certain disease. Journal of Infectious Diseases 35, 423-478.

LyNT, R. K., Jr, Solomon, H. M. \& KaUtTeR, D. A. (1972). Specificity of somatic antisera for detection of Clostridium botulinum by immunofluorescence. In Spores V, pp. 344-351. Edited by H. O. Halvorson, R. Hanson \& L. L. Campbell. Washington, D.C.: American Society for Microbiology.

McClung, L. S. \& ToABe, R. (1947). The egg-yolk reaction for the presumptive diagnosis of Clostridium sporogenes and certain species of the gangrene and botulinum group. Journal of Bacteriology 53, 139-147.

McIntosh, J. (1917). The classification and study of the anaerobic bacteria of war wounds. Medical Research Council Special Report Series, No. 12. London: His Majesty's Stationery Office. 
de Man, J. C., Rogosa, M. \& Sharpe, M. E. (1960). A medium for the cultivation of lactobacilli. Journal of Applied Bacteriology 23, 130-135.

Marie, A. P. (1925). Sur un bacille anaerobie isole d'une viande putrefiee. Comptes rendus des séances de la Société de biologie 93, 21-23.

MetChNikoff, M. E. (1908). Etude sur la flore intestinale. IV. Le Bacillus sporogenes. Annales de l'Institut Pasteur 22, 942-946.

MousSA, R. S. (1956). The relationship between Clostridium septicum and Clostridium chauvoei. Ph.D. thesis, University of Leeds:

Nakamura, S., OKado, I., Nakashio, S. \& Nishida, S. (1977). Clostridium sporogenes isolates and their relationship to $C$. botulinum based on deoxyribonucleic acid reassociation. Journal of General Microbiology 100, 395-401.
Prévot, A. R. (1938). Études de systematique bacterienne. IV. Critique de la conception actuelle du genre Clostridium. Sous-Genre VII (Proteolytique pathogenes) $\mathrm{A}$ - Especes gazeuses putrides. Annales de l'Institut Pasteur 61, 72-91.

PrincewILl, T. J. T. (1969). Deoxyribonucleases and hyaluronidases of Clostridium septicum and Clostridium chauvoei. Fellowship thesis, University of Leeds.

REED, G. B. (1945). Clostridium parasporogenes, an invalid species. Journal of Bacteriology 49, 503505.

Robertson, M. (1918). Notes on Vibrion septique. British Medical Journal 1, 583.

Sturges, W. S. \& Reddish, G. F. (1925). Clostridium flabelliferum: a putrefactive anaerobe with brushlike sporangia. Journal of Bacteriology 11, 37-42. 